\title{
Estudo preliminar teórico de filtro compressor de pulso totalmente fotônico
}

\section{Preliminary theoretical study of the fully photonic pulse compressor filter}

André Paim Gonçalves ${ }^{1, *}$, Renan Miranda Richter ${ }^{1}$, Felipe Streitenberger Ivo ${ }^{1}$, Alessandro Roberto Santos ${ }^{1}$, Robson Ribeiro Carreira ${ }^{1}$, Olympio Lucchini Coutinho'

\section{RESUMO}

Este artigo propôs um estudo breve e teórico para verificar a possibilidade de uso de laços dispersivos com a técnica de autohomodinagem óptica para realizar compressão de pulso linearmente modulado em frequência de radares (linear frequency modulatedLFM). O dispositivo proposto para estudo pretendeu superar as limitações dos dispositivos equivalentes implementados pela eletrônica tal como o filtro baseado em propagação de ondas acústicas de superfície. Essas restrições foram em relação à largura de banda de radiofrequência, frequência da portadora do sinal radar e largura de pulso. Foi feita uma modelagem numérica do dispositivo proposto. Os resultados numéricos indicaram a possibilidade de emprego do compressor de pulso fotônico em radares que estão sendo desenvolvidos com grande produto da largura de banda pela largura do pulso (time bandwidth - TBW).

Palavras-chave: Compressão de pulso, Laço dispersivo, Microwave photonics.

\section{ABSTRACT}

This article proposed a brief and theoretical study to verify the possibility of using dispersive loops with the technique of optic auto homodyne detection to perform linear frequency modulated pulse (LFM) compression in radar signals. The device proposed for study intended to overcome the limitations of equivalent devices implemented by electronics such as the filter based on propagation of surface acoustic waves. These restrictions were in relation to radio frequency bandwidth, radar signal carrier frequency range and pulse width. $\mathrm{A}$ numerical modeling of the device was developed. The numerical results indicated the possibility of using the photonic pulse compressor at radars that are being developed with great product of the bandwidth by pulse width (Time Bandwidth - TBW).

Keywords: Pulse compression, Dispersive loop, Microwave photonics. 


\section{INTRODUÇÃO}

A técnica de compressão de sinais pulsados linearmente modulados em frequência de radares (linear frequency modulated - LFM) de radiofrequência (RF), com o uso de meio dispersivo de propagação, tem sido amplamente utilizada nos sistemas de radares modernos para melhorar a resolução de seus alcances ${ }^{1}$. A compressão de pulso é implementada com o auxílio de um filtro casado. Esse filtro opera como uma linha de atraso empregando surface acoustic wave (SAW). As componentes espectrais do sinal LFM sofrem retardos seletivos, isto é, a que foi gerada primeiramente sofre menor retardo e a última recebe o maior retardo, de modo que todas cheguem ao mesmo tempo à saída da linha de retardo².

A filtragem feita empregando SAW garante otimização da relação sinal ruído (SNR), tornando o receptor robusto a ruídos, e a melhor resolução espacial do pulso. Um filtro típico que emprega linhas de retardo tem limitações, tais como um pico de correlação de banda larga e baixa supressão de lóbulo lateral ${ }^{1,2}$. Por conseguinte, é desejável encontrar um filtro para compressão de pulsos de micro-ondas que ultrapassem essas limitações, bem como a limitação de banda de RF que estes têm. Por outro lado, na atual fase de desenvolvimento de sistemas de radar de banda larga, uma frequência central com dezenas ou mesmo centenas de GHz é muitas vezes necessária ${ }^{1,2}$. Por causa da limitação da largura de banda dos circuitos eletrônicos, o processamento de pulsos de micro-ondas LFM com alta largura de banda de frequência e grande duração é uma tarefa difícili ${ }^{3,4}$.

A métrica chamada de produto time bandwidth (TBW) é muito utilizada para inferir a capacidade de resolução espacial de um radar.

Com o avanço da tecnologia baseada na eletrônica digital, o processamento de sinais de micro-ondas que utilizam técnicas de processamento digital de sinais (DSP) tem demonstrado grande evolução ${ }^{5,6}$, mas o desempenho ainda é limitado em razão da baixa velocidade de amostragem em relação às bandas de frequências necessárias em radares modernos.

A microwave photonics (MWP) surgiu nos anos 70 do século passado. Ela é uma área do conhecimento humano que emprega técnicas de fotônica para gerar, transmitir e processar sinais de RF. A MWP visa superar algumas limitações sofridas pela eletrônica quanto à banda de RF, às portadoras de RF maiores, ao volume, ao peso, ao consumo de energia e à velocidade de resposta ${ }^{7}$.

O intuito deste trabalho foi apontar as possibilidades e limitações do emprego de MWP para resolver o problema de compressão de pulsos de sinais de RF com modulação linear de frequência. O sistema proposto emprega um laço dispersivo e conversão de fase em intensidade somente de uma banda lateral do sinal óptico modulado pelo sinal de interesse. Essa conversão é conhecida com auto-homodinagem óptica ${ }^{8}$. A vantagem de utilizar o laço dispersivo é gerar um efeito multiplicativo na dispersão óptica. A autohomodinagem permite a saída do sistema somente do sinal que sofreu a compressão na banda base. Para verificar a viabilidade desse sistema, foi feita uma modelagem numérica do funcionamento e de suas limitações.

\section{MODELAGEM MATEMÁTICA DO COMPORTAMENTO DO COMPRESSOR DE PULSOS TOTALMENTE FOTÔNICO}

Um sinal óptico gerado por um diodo laser com o campo elétrico igual à Eq. 1:

$$
\vec{E}(t)_{O}=E_{O} \exp \left[j\left(\omega_{O} t+\varphi_{i}\right)\right] \hat{x}
$$

em que: $E_{O}=$ a amplitude de campo elétrico do sinal proveniente do diodo laser; $\omega_{O}=$ sua frequência angular; $t=\mathrm{o}$ tempo; $\varphi_{i}=\mathrm{a}$ fase, incidindo em um modulador de fase.

Visando facilitar a análise do campo $\vec{E}(t)_{O}$, levam-se em conta a fase $\varphi_{i}=0$ e sua orientação coincidente com a do corte que apresenta a melhor resposta do cristal de LiNO3 do modulador de fase, bem como do eixo cartesiano $\hat{x}$.

Considerando o sinal elétrico de RF, $\vec{E}(t)_{R F}$, incidindo na entrada de RF de um modulador de fase, ele é definido como a Eq. 2:

$$
\vec{E}(t)_{R F}=E_{R F} \operatorname{sen}\left(\omega_{R F} t+\varphi_{R F}(t)\right) \operatorname{Rect}\left[\frac{t}{T}\right] \hat{x}
$$

em que: $E_{R F}=$ a amplitude do campo elétrico do sinal de RF incidente; $\omega_{R F}=$ sua frequência angular; $t=\mathrm{o}$ tempo.

Seu valor está compreendido em $-\mathrm{T} / 2<\mathrm{t}<\mathrm{T} / 2$, isto é, na duração entre o sinal de $\mathrm{RF}$ e a fase do sinal de RF que varia no tempo, sendo $\varphi_{R F}(t)=\mu t^{2}$. O campo elétrico é considerado orientado no eixo $\mathrm{x}$, coincidente com a orientação do cristal de $\mathrm{LiNO}_{3}$ do modulador de fase.

No modulador de fase, o sinal óptico sofre uma alteração de fase na forma apresentada pela Eq. 3:

$$
\vec{E}(t)_{O}=E_{O} \exp \left[j \omega_{O} t\right] \times \exp \left[j m \operatorname{sen}\left(\omega_{R F} t+\varphi_{R F}(t)\right) \operatorname{Rect}\left[\frac{t}{T}\right]\right] \hat{x}
$$

Sabendo-se que, conforme a Eq. 4:

$$
V=\frac{E}{d}
$$


em que: $\mathrm{V}=$ o potencial elétrico; $\mathrm{E}$ = campo elétrico; $\mathrm{d}$ = a distância entre os eletrodos do cristal de $\mathrm{LiNO}_{3}$ do modulador de fase.

O índice de modulação m é definido pela Eq. 5:

$$
m=\frac{V}{V_{\pi}} \pi
$$

sendo: $V_{\pi}=$ a tensão de meia onda do modulador de fase.

Reescrevendo a Eq. 3 de forma simplificada em função das séries de Bessel com base nas Eqs. 4, 5 e 6 e considerando a condição especial de operação do modulador conhecida como regime de operação de pequenos sinais, em que $\mathrm{m}<<1$, tem-se a Eq. 7. Dessa forma, o sinal de saída do modulador no regime de operação de pequenos sinais pode ser expresso pelas Eqs. 6 e 7:

$$
\begin{gathered}
\vec{E}(t)_{M}=\operatorname{Rect}\left[\frac{t}{T}\right]\left\{\sum_{i=-\infty}^{i=\infty} E_{O} J_{i}(m) \exp \left[j\left(\omega_{O} t+i\left(\omega_{R F} t+\varphi_{R F}(t)\right)\right)\right]\right\} \hat{x} \\
\vec{E}(t)_{M} \cong \operatorname{Rect}\left\lceil\frac{t}{T}\right\rceil\left\{E_{O} J_{0}(m) \exp \left[j\left(\omega_{O} t\right)\right]+E_{0} J_{1}(m) \exp \left[j\left(\left(\omega_{\bmod } t+\varphi_{R F}(t)\right)\right)\right]-E_{0} J_{1}(m) \exp \left[j\left(\left(\omega_{\text {mod }} t-\varphi_{R F}(t)\right)\right)\right]\right\} \hat{x}
\end{gathered}
$$

em que: $J_{0}(m)$ e $J_{1}(m)$ as funções de Bessel de primeira espécie e ordem 0 e 1 , respectivamente, sabendo-se $\omega_{\text {mod }}=\omega_{O}+\omega_{R F}$.

O sinal óptico na saída do modulador de fase apresenta as bandas laterais iguais em amplitude e simétricas em fase, o que ocasiona um sinal direct current (DC) na saída do fotodetector se as componentes espectrais não sofrerem mudanças na fase ou na amplitude. Esse sinal de saída do modulador de fase é então acoplado a uma grade de Bragg uniforme (FBG) em fibra óptica, conforme Fig. 1, e sintonizada no ponto de operação da grade, indicado na Fig. 2.

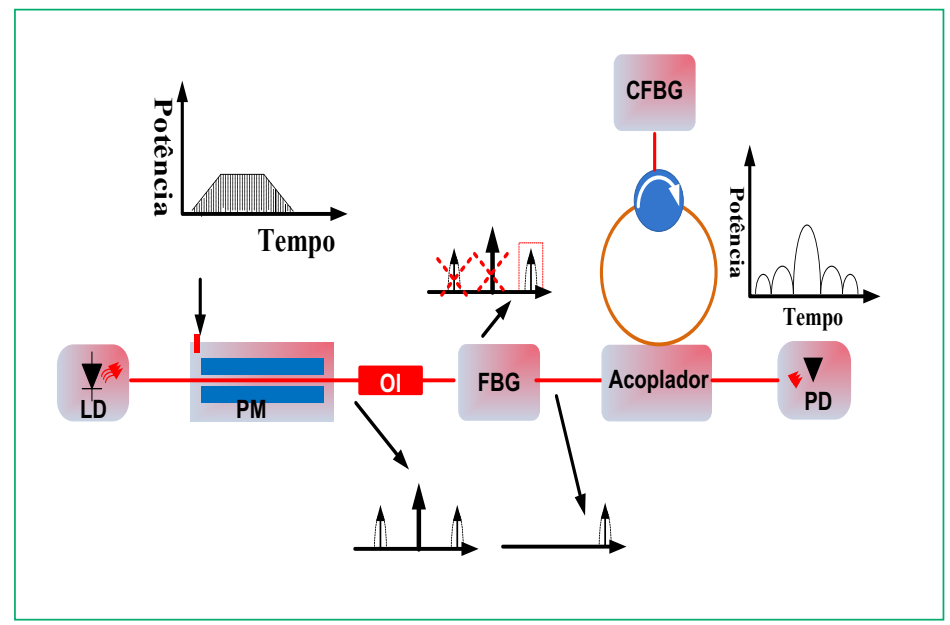

Figura 1: Compressor de pulso totalmente fotônico.

LD: diodo laser; PM: modulador de fase; OI: isolador óptico;

FBG: grade de Bragg uniforme; CFBG: grade de Bragg com chirp linear

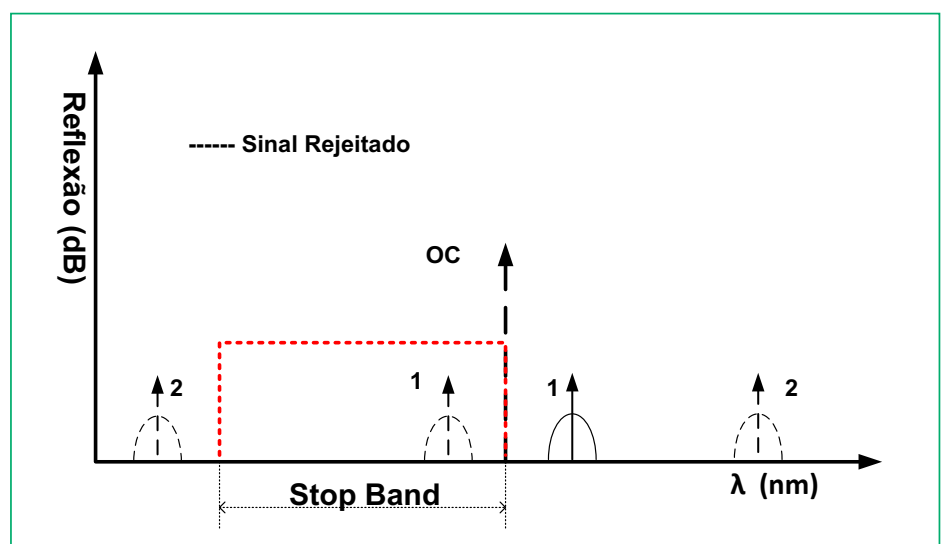

Figura 2: Comportamento de uma grade de Bragg uniforme com a stop band e com o seu limite de operação em torno da banda do sinal de RF (de cor vermelha). Os fasores em tracejado serão descartados. Somente o fasor número 1 de traço contínuo, que representa a banda lateral superior do sinal modulado da portadora óptica com o sinal de RF, será acoplado no laço dispersivo.

oc: optical carrier. 
A sintonia ocorre quando o fasor da portadora óptica fica posicionado no limite superior da curva de refletividade da FBG ideal (stop band), representado pela função degrau (tracejada de cor vermelha). Os fasores que apresentam comprimento de ondas compreendidos na stop band serão refletidos e os demais transmitidos. Os fasores com o numeral 2 não sofrerão conversão de modulação de fase em intensidade, pois estes não sofreram mudanças de fase nem de amplitude suficientes para essa conversão.

O sinal óptico quando se propaga na FBG tem a portadora e uma de suas bandas laterais refletidas, conforme a Fig. 2, e a outra banda lateral é transmitida. Esta última banda lateral será acoplada no laço dispersivo e no fotodetector. Faz-se importante observar que a FBG uniforme é projetada para possuir alto coeficiente de refletividade óptica, sendo praticamente constante para a faixa de frequência equivalente à de RF na região da stop band.

A sintonia do filtro óptico formado pela grade de Bragg é feita de modo que a frequência angular do diodo laser $\omega_{O}$ assuma um valor tal que, subtraído do sinal de RF, $\omega_{R F}$, fique na faixa espectral de refletividade máxima da grade, conforme a Fig. 2. Essa sintonia fará com que somente a banda lateral superior do sinal incida no laço dispersivo e no fotodetector. Seu campo elétrico é expresso pela Eq. 8:

$$
\vec{E}(t)_{T} \cong t(\omega) E_{1} J_{1}(m) \times\left\{\exp \left[j\left(\left(\omega_{\text {mod }} t+\varphi_{R F}(t)\right)+\varphi(\omega)\right)\right]\right\} \operatorname{Rect}\left[\frac{t}{T}\right] \hat{x}
$$

Em que o campo elétrico depende diretamente da resposta em frequência dos coeficientes de transmissão, $t(\omega)=1-\mathrm{r}(\omega)$, e fase, $\varphi(\omega)$, da FBG. Relembra-se que a parcela do sinal referente à portadora óptica e à banda lateral inferior foi filtrada, isto é, $t \omega_{O}=t\left(\omega_{O}-\omega_{R F}\right)=0$.

Observando que a grade de Bragg tem o comportamento uniforme, consideram-se $=\varphi\left(\omega_{O}+\omega_{R F}\right)=0$ e $t\left(\omega_{O}+\omega_{R F}\right)=\Gamma$. Reescrevendo-se a Eq. 8, tem-se a Eq. 9:

$$
\vec{E}(t)_{T} \cong \Gamma E_{1} J_{1}(m) \times\left\{\exp \left[j\left(\omega_{\bmod } t+\varphi_{R F}(t)\right)\right]\right\} \operatorname{Rect}\left[\frac{t}{T}\right] \hat{x}
$$

O sinal óptico, formado da banda superior da modulação do sinal de RF, incide no acoplador direcional e sofre uma divisão. Uma parcela acopla no outro guia do acoplador que se propaga em loop dispersivo composto de dispositivo com grande dispersão. Esse sistema com o loop deverá ter um efeito multiplicativo da dispersão. Isso se deve ao fato de o sinal perfazer esse laço $n$ vezes ${ }^{2}$. As perdas por inserção, amplificação e outras mais estão definidas no fator g na Eq. 10. O sinal que não acoplou no segundo guia do acoplador direcional incide no fotodetector sem ser comprimido no loop dispersivo. Como o sinal está modulado em fase e não foi convertido de modulação de fase para intensidade por causa da dispersão do loop, este por ocasião da saída no fotodetector será um sinal DC.

A resposta modelada matematicamente do laço dispersivo ao impulso na sua entrada no domínio da frequência fica em conformidade com as Eqs. 10, 11, 12 e 13:

$$
\begin{gathered}
H(\omega) \cong g^{n} \exp \left(j n \Phi_{0}\right) \exp \left(j n \Phi_{1}\left(\omega-\omega_{\text {mod }}\right)\right) \exp \left(j \frac{n \Phi_{2}}{2}\left(\omega-\omega_{\text {mod }}\right)^{2}\right) \\
\Phi_{0}=\varphi(\omega) \\
\Phi_{1}=\frac{\partial(\varphi(\omega))}{\partial \omega} \\
\Phi_{2}=\frac{\partial^{2}(\varphi(\omega))}{\partial \omega^{2}}
\end{gathered}
$$

em que: $\Phi_{0}=$ a fase que representa o atraso do sinal (rad); $\Phi_{1}=$ a fase que representa o atraso de grupo do sinal (s); $\Phi_{2}=$ a fase que representa a dispersão de atraso de grupo $\left(\mathrm{s}^{2} / \mathrm{rad}\right)$.

Transformando a resposta do loop do impulso do domínio da frequência no domínio do tempo conforme Eq. 149:

$$
h(t)=\int_{-\infty}^{\infty} H(\omega) \exp (j \omega t) d \omega
$$

Obtém-se a Eq. 15:

$$
\mathrm{h}(\mathrm{t})=\int_{-\infty}^{\infty} g^{n} \exp \left(j n \Phi_{0}\right) \exp \left(j n \Phi_{1}\left(\omega-\omega_{\bmod }\right)\right) \exp \left(j \frac{n \Phi_{2}}{2}\left(\omega-\omega_{\bmod }\right)^{2}\right) \exp (\mathrm{j} \omega \mathrm{t}) \mathrm{d} \omega
$$

Desenvolvendo a Eq. 15, tem-se a Eq. 16:

$h(t)=g^{n} \exp \left(j n \Phi_{0}\right) \exp \left(-j n \Phi_{1} \omega_{\text {mod }}\right) \exp \left(j \frac{n \Phi_{2} \omega_{m o d}^{2}}{2}\right) \int_{-\infty}^{\infty} \exp \left(j n \Phi_{1} \omega\right) \exp \left(j \frac{n \Phi_{2}}{2}\left(-2 \omega \omega_{\bmod }+\omega^{2}\right)\right) \exp (j \omega t) d \omega$ 
Rearranjando os argumentos das fases que estão dentro da integral, tem-se a Eq. 17:

$$
h(t)=g^{n} \exp \left(j n \Phi_{0}\right) \exp \left(-j n \Phi_{1} \omega_{\bmod }\right) \exp \left(j \frac{n \Phi_{2} \omega_{m o d}^{2}}{2}\right) \int_{-\infty}^{\infty} \exp \left(j \omega\left(n \Phi_{1}+t-n \Phi_{2} \omega_{\bmod }\right)\right) \exp \left(j \frac{n \Phi_{2} \omega^{2}}{2}\right) d \omega
$$

Visando facilitar a visualização da expressão e sua evolução matemática, substituíram-se os termos que estão fora da integral pela variável muda c, conforme Eqs. 18 e 19:

$$
\begin{gathered}
c=g^{n} \exp \left(j n \Phi_{0}\right) \exp \left(-j n \Phi_{1} \omega_{\text {mod }}\right) \exp \left(j \frac{n \Phi_{2} \omega_{\text {mod }}^{2}}{2}\right) \\
h(t)=c \int_{-\infty}^{\infty} \exp \left(j \omega\left(n \Phi_{1}+t-n \Phi_{2} \omega_{\text {mod }}\right)\right) \exp \left(j \frac{n \Phi_{2} \omega^{2}}{2}\right) d \omega
\end{gathered}
$$

Observando-se a Eq. 19 e sabendo-se que sua integral pode ter seu resultado obtido pela Eq. 20, aplica-se o recurso de substituição de variável dos termos que multiplicam $\omega$ e $\omega^{2}$, respectivamente, conforme Eqs. 21 e 22, e, sabendo-se da propriedade matemática expressa na Eq. 23, obtém-se a Eq. 24.

$$
\begin{gathered}
\int_{-\infty}^{\infty} \exp \left(-a \omega^{2}-b \omega\right) d \omega=\sqrt{\frac{\pi}{a}} \exp \frac{b^{2}}{4 a} \\
a=-j \frac{n \Phi_{2}}{2} \\
b=-j\left(n \Phi_{1}+t-n \Phi_{2} \omega_{\text {mod }}\right) \\
b^{2}=(x+y-z)^{2}=x^{2}+y^{2}+z^{2}+2 x y-2 x z-2 y z \\
b^{2}=\left(\mathrm{n}^{2} \Phi_{1}^{2}+\mathrm{t}^{2}+\mathrm{n}^{2} \Phi_{2}^{2} \omega_{\text {mod }}^{2}+2 \mathrm{n} \Phi_{1} \mathrm{t}-2 \mathrm{n}^{2} \Phi_{1} \Phi_{2} \omega_{\text {mod }}-2 \mathrm{n} \Phi_{2} \omega_{\text {mod }} \mathrm{t}\right)
\end{gathered}
$$

Reorganizando a Eq. 24 para facilitar a aplicação dos resultados obtidos nas Eqs. 25, 26 e 27, conforme:

$$
\begin{gathered}
b^{2}=\left(\mathrm{n}^{2} \Phi_{1}^{2}+\mathrm{n}^{2} \Phi_{2}^{2} \omega_{\mathrm{mod}}^{2}-2 \mathrm{n}^{2} \Phi_{1} \Phi_{2} \omega_{m o d}+2 \mathrm{n} \Phi_{1} \mathrm{t}-2 \mathrm{n} \Phi_{2} \omega_{\bmod } \mathrm{t}+\mathrm{t}^{2}\right) \\
4 a=-j \frac{n \Phi_{2}}{2}=-2 j n \Phi_{2} \\
\frac{b^{2}}{4 a}=\frac{\left(\mathrm{n}^{2} \Phi_{1}^{2}+\mathrm{n}^{2} \Phi_{2}^{2} \omega_{\bmod }^{2}-2 \mathrm{n}^{2} \Phi_{1} \Phi_{2} \omega_{m o d}+2 \mathrm{n} \Phi_{1} \mathrm{t}-2 \mathrm{n} \Phi_{2} \omega_{m o d} \mathrm{t}+\mathrm{t}^{2}\right)}{-2 j n \Phi_{2}}
\end{gathered}
$$

Com a simplificação da Eq. 27, obtém-se a Eq. 28, conforme:

$$
\frac{b^{2}}{4 a}=\mathrm{j}\left(\mathrm{n} \frac{\Phi_{1}^{2}}{2 \Phi_{2}}+\mathrm{n} \frac{\Phi_{2} \omega_{\bmod }^{2}}{2}-\mathrm{n} \Phi_{1} \omega_{\bmod }+\frac{\Phi_{1} \mathrm{t}}{\Phi_{2}}-\omega_{\bmod } \mathrm{t}+\frac{\mathrm{t}^{2}}{2 \mathrm{n} \Phi_{2}}\right)
$$

Aplicando-se o valor obtido na Eq. 21 na primeira parcela do lado direito da igualdade na Eq. 20, tem-se a Eq. 29:

$$
\sqrt{\frac{\pi}{a}}=\sqrt{\frac{\pi}{-j \frac{n \Phi_{2}}{2}}}=\sqrt{\frac{2 \mathrm{j} \pi}{n \Phi_{2}}}=\sqrt{\frac{2 \pi}{n \Phi_{2}}} \sqrt{\mathrm{j}}=\sqrt{\frac{2 \pi}{n \Phi_{2}}} \exp \left(\frac{\pi}{4}\right)
$$

Realizando a substituição de variável nas parcelas que multiplicam te os termos que não são multiplicados pelo tempo, respectivamente, encontram-se as Eqs. 30 e 31, conforme:

$$
\begin{gathered}
\beta\left(\Phi_{2}\right)=\beta=\Phi_{1}-\Phi_{2} \omega_{\text {mod }} \\
\gamma\left(\Phi_{2}\right)=\gamma=\sqrt{\frac{2 \pi}{n \Phi_{2}}} \operatorname{expj}\left(\frac{\pi}{4}+\mathrm{n} \frac{\Phi_{1}^{2}}{2 \Phi_{2}}+\frac{\mathrm{n} \Phi_{2} \omega_{m o d}^{2}}{2}-n \Phi_{1} \omega_{m o d}\right)
\end{gathered}
$$

Aplicando-se todos os termos calculados em $h(t)$, tem-se a Eq. 32:

$$
h(t)=\operatorname{crexpj}(\beta t) \operatorname{expj}\left(\frac{-t^{2}}{2 n \Phi_{2}}\right)
$$


O sinal óptico na saída do loop dispersivo da Eq. 9 e o sinal representado pela Eq. 32 aplicados na Eq. 33 resulta na Eq. 34, na qual este último é o resultado da convolução das Eqs. 9 e 32, conforme Eq. 33:

$$
\begin{gathered}
\left|\vec{E}(t)_{S}\right|=\left|\vec{E}(t)_{T}\right| * h(t) \\
\left|\vec{E}(t)_{S}\right|=\int_{-\infty}^{\infty} \operatorname{Rect}\left[\frac{t}{T}\right] \Gamma E_{1} J_{1}(m) \exp \left[j\left(\left(\omega_{\bmod }+\varphi_{R F}(t)\right) t\right)\right] \cdot \operatorname{c\gamma expj}\left(\frac{\beta(t-\tau)}{\Phi_{2}}\right) \operatorname{expj}\left(\frac{-(t-\tau)^{2}}{2 n \Phi_{2}}\right) d \tau
\end{gathered}
$$

Reorganizando a Eq. 34 com o posicionamento dos termos que não dependem de $t$ para fora da integral e ajustando o intervalo da integral para a duração do sinal definido pela função $\operatorname{Rect}\left[\frac{t}{T}\right]$, em que ela vale 1 para o intervalo $-\mathrm{T} / 2<\mathrm{t}<\mathrm{T} / 2$, obtém-se a Eq. 35 :

$$
\left|\vec{E}(t)_{S}\right|=c \gamma \Gamma E_{1} J_{1}(m) \operatorname{expj}\left(\frac{\beta t}{\Phi_{2}}\right) \operatorname{expj}\left(\frac{-t^{2}}{2 n \Phi_{2}}\right) \int_{-}^{T} T / 2 \exp \left[j\left(\omega_{m o d} \tau+\mu \tau^{2}\right)\right] \operatorname{expj}\left(\frac{\beta \tau}{\Phi_{2}}\right) \operatorname{expj}\left(\frac{-t^{2}+2 t \tau}{2 n \Phi_{2}}\right) d \tau
$$

Substituindo-se a Eq. 30 pela Eq. 35, tem-se a Eq. 36:

$$
\left|\vec{E}(t)_{S}\right|=\mathrm{c} \gamma \Gamma E_{1} J_{1}(m) \operatorname{expj}\left(\frac{\beta t}{\Phi_{2}}\right) \operatorname{expj}\left(\frac{-t^{2}}{2 n \Phi_{2}}\right) \int_{-T / 2}^{T / 2} \exp \left[j\left(\omega_{\bmod } \tau+\mu \tau^{2}\right)\right] \operatorname{expj}\left(\frac{\left(\Phi_{1}-\Phi_{2} \omega_{m o d}\right) \tau}{\Phi_{2}}\right) \operatorname{expj}\left(\frac{-\tau^{2}}{2 n \Phi_{2}}+\frac{t \tau}{n \Phi_{2}}\right) d \tau
$$

Considerando-se a situação em que os termos que multiplicam $t^{2}$ são iguais ${ }^{10}$, obtém-se a Eq. 37:

$$
\mu=\frac{1}{2 n \Phi_{2}}
$$

Ao atingir a condição da Eq. 37, a expressão da Eq. 36 passa a ser a transformada de Fourier da envoltória do sinal de entrada, definida pela função $\operatorname{Rect}\left[\frac{t}{T}\right]$. Esse fato torna-se mais facilmente observável após a organização da Eq. 38, que se transforma em Eq. 39, e depois com o uso de substituição de variável, conforme Eqs. 40 e 41.

$$
\begin{gathered}
\left|\vec{E}(t)_{S}\right|=c \gamma \Gamma E_{1} J_{1}(m) \operatorname{expj}\left(\frac{\beta t}{\Phi_{2}}\right) \operatorname{expj}\left(\frac{-t^{2}}{2 n \Phi_{2}}\right) \int_{-T / 2}^{T / 2} \operatorname{expj}\left(\frac{\Phi_{1} \tau}{\Phi_{2}}+\frac{t \tau}{n \Phi_{2}}\right) d \tau \\
\left|\vec{E}(t)_{S}\right|=c \gamma \Gamma E_{1} J_{1}(m) \operatorname{expj}\left(\frac{\beta t}{\Phi_{2}}\right) \operatorname{expj}\left(\frac{-t^{2}}{2 n \Phi_{2}}\right) \int_{-T / 2}^{T / 2} \operatorname{expj}\left(\frac{n \Phi_{1}+t}{n \Phi_{2}}\right) d \tau \\
W=\frac{n \Phi_{1}+t}{n \Phi_{2}} \\
\left|\vec{E}(t)_{S}\right|=c \gamma \Gamma E_{1} J_{1}(m) \operatorname{expj}\left(\frac{\beta t}{\Phi_{2}}\right) \operatorname{expj}\left(\frac{-t^{2}}{2 n \Phi_{2}}\right) \int_{-T / 2}^{T / 2} \operatorname{expj} W \tau d \tau
\end{gathered}
$$

Resolvendo a Eq. 41, observa-se a Eq. 42:

$$
\left|\vec{E}(t)_{S}\right|=c \gamma \Gamma E_{1} J_{1}(m) \operatorname{expj}\left(\frac{\beta t}{\Phi_{2}}\right) \operatorname{expj}\left(\frac{-t^{2}}{2 n \Phi_{2}}\right)\left[2 \frac{\operatorname{expj} W(T / 2)-\operatorname{expjW}(-T / 2)}{2 j W}\right]
$$

E obtém-se a Eq. 43:

$$
\left|\vec{E}(t)_{S}\right|=2 \mathrm{c} \gamma \Gamma E_{1} J_{1}(m) \operatorname{expj}\left(\frac{\beta t}{\Phi_{2}}\right) \operatorname{expj}\left(\frac{-t^{2}}{2 n \Phi_{2}}\right) \operatorname{sinc}\left[\frac{n \Phi_{1}+t}{n \Phi_{2}}(T / 2)\right]
$$

Com a reorganização da Eq. 43, é obtido o módulo do campo elétrico, $\left|\vec{E}(t)_{S}\right|$, incidente no fotodetector, conforme Eq. 44:

$$
\left|\vec{E}(t)_{S}\right|=2 \mathrm{c} \gamma \Gamma E_{1} J_{1}(m) \operatorname{expj}\left(\frac{\beta t}{\Phi_{2}}\right) \operatorname{expj}\left(\frac{-t^{2}}{2 n \Phi_{2}}\right) \operatorname{sinc}\left[\frac{\Phi_{1} T}{2 \Phi_{2}}+\frac{\mu T}{2} t\right]
$$

Sabendo-se que a Eq. 45 representa o vetor campo elétrico incidente no fotodetector, conforme:

$$
\vec{E}(t)_{S}=2 \mathrm{c} \gamma \Gamma E_{1} J_{1}(m) \operatorname{expj}\left(\frac{\beta t}{\Phi_{2}}\right) \operatorname{expj}\left(\frac{-t^{2}}{2 n \Phi_{2}}\right) \operatorname{sinc}\left[\frac{\Phi_{1} T}{2 \Phi_{2}}+\frac{\mu T}{2} t\right] \hat{x}
$$

Por meio da Eq. 45, foi possível determinar o vetor campo magnético, $\vec{H}(t)_{S}$, conforme Eq. 46 :

$$
\vec{H}(t)_{S}=\frac{2 c \gamma \Gamma J_{1}(m)}{\eta} \operatorname{expj}\left(\frac{\beta t}{\Phi_{2}}\right) \operatorname{expj}\left(\frac{-t^{2}}{2 n \Phi_{2}}\right) \operatorname{sinc}\left[\frac{\Phi_{1} T}{2 \Phi_{2}}+\frac{\mu T}{2} t\right] \hat{y}
$$


em que: $\eta$ = a impedância intrínseca do meio.

Aplicando-se o conceito do módulo do vetor de Poynting, definido na Eq. 47, as fases que estavam inseridas em c e $\gamma$ desaparecem por conta de ambas ocorrerem em $\vec{E}(t)_{\mathrm{S}}$ e $\vec{H}(t)_{S}^{*}$ e estarem com os seus valores simétricos. Resolvendo a Eq. 47, é possível conhecer a intensidade do sinal óptico incidente no fotodetector representado pela Eq. 48, conforme segue:

$$
\begin{gathered}
|\vec{S}(t)|=\left|\vec{E}(t)_{\mathrm{S}} \times \vec{H}(t)_{S}^{*}\right| \\
|\vec{S}(t)|=\left\{\frac{8 \pi\left(\Gamma E_{1} J_{1}(m)\right)^{2} g^{2 n}}{n \eta \Phi_{2}} \operatorname{sinc}^{2}\left[\frac{\Phi_{1} T}{2 \Phi_{2}}+\frac{\mu T}{2} t\right]\right\}
\end{gathered}
$$

Simplificando a Eq. 48, tem-se a Eq. 49:

$$
|\vec{S}(t)|=\frac{4 \pi\left(\Gamma E_{1} J_{1}(m)\right)^{2} g^{2 n}}{n \eta \Phi_{2}} \operatorname{sinc}^{2}\left[\frac{\Phi_{1} T}{2 \Phi_{2}}+\frac{\mu T}{2} t\right]
$$

A Eq. 49 apresenta uma função $\operatorname{sinc}^{2}$, que representa o quadrado da transformada de Fourier da envoltória do sinal de entrada. Esse resultado demonstra que o sinal na saída estará na banda base, depende de uma fase constante igual a $\frac{\Phi_{1} T}{2 \Phi_{2}}$ e que a largura do sinal depende de $\frac{\mu T^{2}}{2}$, para $\mathrm{t}=\mathrm{T}$ e utilizando a Eq. 37, sendo igual a $\frac{T^{2}}{4 n \Phi_{2}}$. Como $\Phi_{2}<1 \mathrm{e} \mathrm{n}$, com grandes valores, fazem a relação apresentar valores maiores, a função $\operatorname{sinc}^{2}$ reduz sua duração do lóbulo principal. Tal fato indica que a envoltória tem sua largura de pulso reduzida.

O parâmetro dispersão de atraso de grupo, $\Phi_{2}$, pode ser calculado pela Eq. 50 :

$$
\left|\Phi_{2}\right|=\frac{\lambda_{C F B G}^{2} D}{2 \pi c_{l u z}}
$$

em que: $c_{l u z}=$ a velocidade da luz no vácuo; $\mathrm{D}=$ a dispersão óptica, dada em $\mathrm{ps} / \mathrm{nm} ; \lambda_{C F B G}=$ o comprimento de onda central da grade de Bragg com chirp linear.

Os valores de $\mu$ podem ser calculados pelas Eqs. 37 e 50, resultando na Eq. $51^{10}$ :

$$
\mu=\frac{1000 \pi \mathrm{c}_{\mathrm{luz}}}{\lambda_{C F B G}^{2} n D}
$$

Cabe acrescentar que, havendo atraso das componentes espectrais do sinal LFM deslocado da portadora óptica, em que essas componentes incidem ao mesmo tempo no fotodetector, se permite o batimento entre elas. Se o sinal, por acaso, não sofrer o atraso correto para as suas componentes espectrais, não haverá o batimento entre elas. Logo, não sairá o sinal na banda base, e, sim, somente o sinal DC. Mesmo ocorrendo o batimento entre algumas das componentes do sinal LFM, o nível do sinal na banda base após o fotodetector será baixo.

Os valores de dispersão óptica, D, empregados nos cálculos, conforme a Tabela 1, foram retirados de especificações de alguns fabricantes de grades de Bragg. A relação de equivalência entre comprimentos de ondas e frequências é de 0,8 nm para $100 \mathrm{GHz}$, de acordo com a norma da International Telecommunication Union - Telecommunication Standardization Sector (ITU-T) para dispositivos compostos de sílica.

Tabela 1: Resultados dos cálculos da chirp rate $(\mu)$ por meio da Eq. 51.

\begin{tabular}{|c|c|c|}
\hline Chirp rate $(\mathrm{GHz} / \mathbf{n s})$ & Número de voltas & D (ps/nm) \\
\hline 156,8 & 1 & 2.500 \\
\hline 3,14 & 50 & 2.500 \\
\hline 261,4 & 1 & 1.500 \\
\hline 5,23 & 50 & 1.500 \\
\hline
\end{tabular}

O valor de dispersão de $0,4 \mu \mathrm{s} / \mathrm{MHz}=0,4 \times 10^{6} \mathrm{~ns} / \mathrm{GHz}$ é encontrado em dispositivos a SAW e serviu como referência para as comparações ${ }^{11}$. Comparando-se esse valor com os obtidos na Tabela 1, percebe-se que, para detecção de sinais radar típicos encontrados no comércio, esse sistema ainda não poderá ser capaz de realizar compressão de pulso, pois precisaria dar mais voltas. Para dar mais voltas, haveria a necessidade de amplificação óptica com maior controle de ruído inserido no sistema. Em radares com grandes valores de TBW da ordem de 74.000, conforme Herrera et al. ${ }^{4}$, por exemplo, seria possível realizar a compressão de pulso com menor dispersão. A dispersão cromática acumulada no laço dispersivo não consegue ser da ordem de grandeza da largura de pulsos de radares comerciais. Para a largura de pulsos da ordem de nanossegundos, esses pulsos dependem de uma grande chirp rate para manter a TBW grande. 
Quanto maior for a banda abrangida pelo sinal, maior vai ser o efeito da dispersão do dispositivo. Há a necessidade de se estudar a quantidade de voltas que um laço dispersivo consegue manter com e sem a amplificação. A estimativa de empregar um laço que dura 50 voltas foi uma estimativa conservadora baseada em Nguyen et al. ${ }^{12}$.

\section{CONCLUSÃO}

A crescente demanda de banda pelos sistemas de radares modernos e, por conseguinte, portadores de RF maiores, bem como de larguras de pulsos maiores, demonstra que dispositivos que dependem da eletrônica não estão conseguindo superar suas limitações. Os dispositivos baseados em MWP estão surgindo para ajudar a superar as limitações da eletrônica. O sistema proposto neste trabalho mostrou-se com limitações para aplicação em radares atuais, contudo revelou-se uma opção para a detecção de radares com grandes TBW, em que a largura de pulso é pequena e a largura de banda é muito grande. A limitação apresentada neste artigo poderá ser superada com a construção de grades de Bragg com dispersões maiores ou com o uso de materiais mais dispersivos do que a sílica empregada nas grades. Há a necessidade de se estudar a viabilidade de saber quantas voltas um laço dispersivo consegue manter com e sem a amplificação.

\section{REFERENCES}

1. Richards MA, Scheer JA, Holm WA. Principles of modern radar: basic principles. Reino Unido: Institution of Engineering and Technology; 2010.

2. Mahafza BR. Radar systems analysis and design using MATLAB. $3^{\mathrm{a}}$ ed. Boca Raton: CRC Press; 2016.

3. Wang C, Yao J. Large time-bandwidth product microwave arbitrary waveform generation using a spatially discrete chirped fiber bragg grating J Light Technol. 2010;28(11):1652-60. https://doi.org/10.1109/JLT.2010.2047093

4. Herrera LEY, Ribeiro RM, Jabulka VB, von der Weid JP. Optical generation and transmission of linearly chirped microwave pulses with high time-bandwidth product. Optics InfoBase Conference Papers; 2018. https://doi.org/10.1364/OFC.2018.M1H.2

5. Capmany J, Mora J, Gasulla I, Sancho J, Lloret J, Sales S. Microwave photonic signal processing. J Light Technol. 2013;31(4):571-86. https:// doi.org/10.1109/JLT.2012.2222348

6. Ghelfi P, Scotti F, Onori D, Bogoni A. Photonics for Ultrawideband RF Spectral Analysis in Electronic Warfare Applications. IEEE J Sel Top Quantum Electron. 2019;25(4):8900209. https://doi.org/10.1109/JSTQE.2019.2902917

7. Yao J, Zou X, Chen H, Capmany J. Guest Editorial: Microwave photonics. J Light Technol. 2018;36(19):4216-8. https://doi.org/10.1109/ JLT.2018.2864447

8. Hossein-Zadeh M, Levi AFJ. Selfhomodyne photonic microwave receiver architecture based on linear optical modulation and filtering. Microw Opt Technol Lett. 2008;50(2):345-50. https://doi.org/10.1002/mop.23065

9. Caloz C, Gupta S, Zhang Q, Nikfal B. Analog Signal Processing: A Possible Alternative or Complement to Dominantly Digital Radio Schemes. IEEE Microw Mag. 2013;14(6):87-103. https://doi.org/10.1109/mmm.2013.2269862

10. Song C, Qian J, Lei M, Zheng Z, Huang S, Gao X. A chirp-rate-tunable microwave photonic pulse compression system for multi-octave linearly chirped microwave waveform. IEEE Photonics J. 2019;11(2):5501113. https://doi.org/10.1109/JPHOT.2019.2897743

11. Webster JG. Encyclopedia of Electrical and Electronics Engineering. Nova York: Wiley; 2014.

12. Nguyen TA, Chan EHW, Minasian RA. Photonic multiple frequency measurement using a frequency shifting recirculating delay line structure. J Light Technol. 2014;32(20):3831-8. https://doi.org/10.1109/JLT.2014.2329488 